\title{
Comparison of Pre-, Post- and Symmetrical-Dispersion Compensation Schemes for 10/15Gbps using Different Modulation Formats at Various Optical Power Levels using Standard and Dispersion Compensated Fibers
}

\author{
Rajani \\ Dept. of Electronics \& \\ Communication Engineering \\ SBSCET, Firozpur \\ Punjab, India
}

\author{
Raju Pal \\ Dept. of Computer Science \& \\ Engineering \\ National Institute of \\ TechnologyJalandhar, Punjab,
}

\author{
Vishal Sharma \\ Dept. of Electronics \& \\ Communication Engineering \\ SBSCET, Firozpur
}

\begin{abstract}
In this paper, we investigate pre-, post- and symmetricaldispersion compensation methods for $10 / 15 \mathrm{~Gb} / \mathrm{s}$ using different modulation formats like NRZ, RZ and RZ Super gaussian using standard and dispersion compensated fibers through computer simulations to optimize high data rate optical transmission. The influence of EDFA power of fiber has been studied to evaluate the performance of optical communication systems. The performance characteristics like bit error rate, Q parameter, required optical power at the output are studied by simulating different systems. The influence of variation in length of fiber of each span with the variation of power of EDFA is also studied, and conclude that post and symmetric scheme is better.
\end{abstract}

\section{General Terms}

Optical Fiber Compensation, Dispersion Compensation Technique.

\section{Keywords}

Optical Communication systems, DCF compensation Techniques, Dispersion, High Speed Optical Communication System.

\section{INTRODUCTION}

Recently, there has been great interest in using single mode fibers [1] for high-bit-rate transmission in low loss transmission windows but dispersion is an important impairment that degrades overall system performance of an optical communication system. At high-bit-rate, the dispersion-induced broadening of short pulses propagating in the fiber causes crosstalk between the adjacent time slots, leading to errors when the communication distance increases beyond the dispersion length of the fiber [2].The advent of erbium-doped amplifiers (EDFAs) operating in the $1.55 \mathrm{~lm}$ region has increased the link distance as limited by fiber loss in optical communication systems. How ever, these amplifiers induce nonlinear effects, which not only limit the bit rate but also the propagation distance in an optical fiber link. Nonlinear effects along with dispersion are the destructive forces for pulse propagation in ultra high-bit-rate optical transmission system [3] and cause power penalty and other impairments in the system. Therefore e, in order to realize the high data rates over long distances down the SM fiber, techniques must be found to overcome signal degradation due to dispersion and nonlinearities .Several methods have been proposed to overcome the impairments caused by chromatic dispersion including initial pre-chirp [4], microchip compensation [5], mid span spectral inversion [6], optical phase conjugation [7-9], dispersion-supported transmission [10], dispersion compensating devices [11-13] and differential delay method [14-18]. The use of dispersion compensated fiber is an important method for dispersion compensation and to upgrade the already installed links of single mode fiber [19].Dispersion compensated fibers are specially designed fibers with negative dispersion. The high value of negative dispersion is used to compensate for positive dispersion over large lengths of ordinary fiber. The total negative dispersion compensates for the total positive dispersion. Spans made of single mode fibers and dispersion compensated fibers are good candidates for long distance transmission as their high local dispersion is known to reduce the phase matching giving rise to four wave mixing in wavelength division multiplexed (WDM) systems .Signal degradation in such systems is due to combined effects of group velocity dispersion, kerr non-linearity and accumulation of amplified spontaneous emission due to periodic amplification. Compensation is done by three methods, pre -, post - and symmetrical compensation. Here in this paper we investigate the behavior of pre ,post and symmetric compensation techniques on the bases of two cases: (1) when the length of the fibers is fixed and power of EDFA is varied. (2) when both the length of the fiber of each span and power or EDFA is varied. On the bases of some parameters like bit error rate, Q parameter, and required optical power, using different modulation formats.

\section{SIMULATION SETUP}

For simulation of pre-, post- and symmetrical-DCF schemes, each transmitter section consists of data source of different formats such as NRZ, RZ and RZ super-Gaussian at different bit rate of 10- and 15-Gbps, electrical driver to generate the desired data transmission format by converting the logical input signal into an electrical signal, the laser source line width is $100 \mathrm{MHz}$ amplitude modulator. In the first case, the optical communication system is pre-compensated by the dispersion compensated fiber of negative dispersion of $80 \mathrm{ps} / \mathrm{nm} / \mathrm{km}$ of proportionate length, calculated as $\mathrm{L}_{\mathrm{DCF}} / \mathrm{L}_{\mathrm{SMF}}$ $\sim 1 / 6$, against the standard fiber of $16 \mathrm{ps} / \mathrm{nm} / \mathrm{km}$. In the second case, the optical communication system is post-compensated by the dispersion compensated fiber negative dispersion of $80 \mathrm{ps} / \mathrm{nm} / \mathrm{km}$ of proportionate length against standard fiber of $16 \mathrm{ps} / \mathrm{nm} / \mathrm{km}$. In the third case of symmetrical compensation configuration, the system is symmetrically compensated by two dispersion compensated fibers negative dispersion of $80 \mathrm{ps} / \mathrm{nm} / \mathrm{km}$ of proportionate length against standard fiber of in between with EDFA amplifiers after each type of fiber. The 
output is detected at the receiver by PIN detector and is passed through electrical filter and output is observed on electroscope.

\section{RESULT\& DISCUSSION}

In this paper we investigate the behaviour of pre-, post- and symmetric compensation techniques on the bases of two cases given below and conclude, which compensation is perform better for each case. The parameters like bit error rate, value of $\mathrm{Q}$ and required optical power is measured for each case, at $10 / 15 \mathrm{Gbps}$ while laser line width of laser diode is $100 \mathrm{MHz}$

\subsection{When length of fibre of each span is fixed}

\subsubsection{Q Parameter Evaluation}

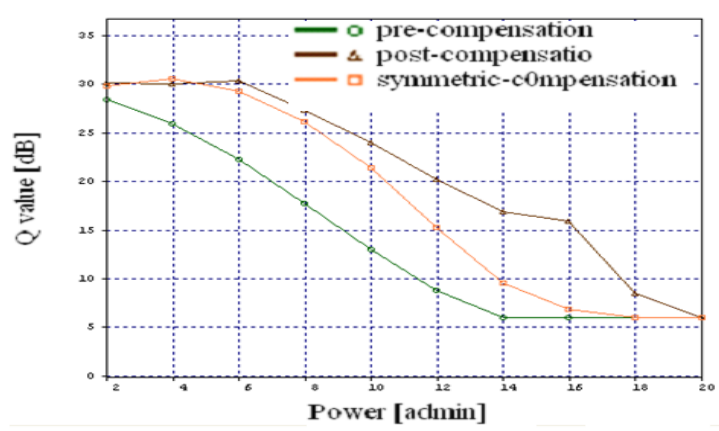

(a)

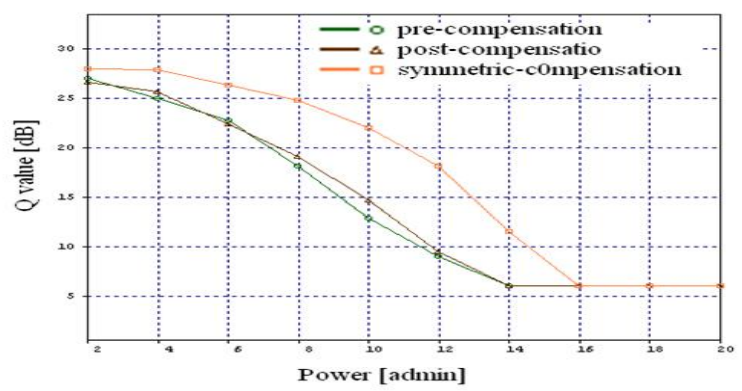

(b)

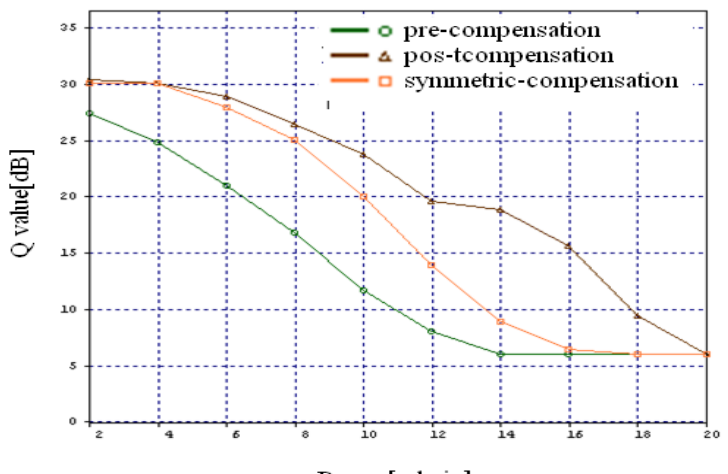

(c)

Fig 1: Q-parameter Vs power of EDFA with $100 \mathrm{MHz}$ Laser Line width using different DCF compensation Techniques with (a) NRZ Format (b) RZ Format and (c) RZ-Super Gaussian Format at 10Gbps

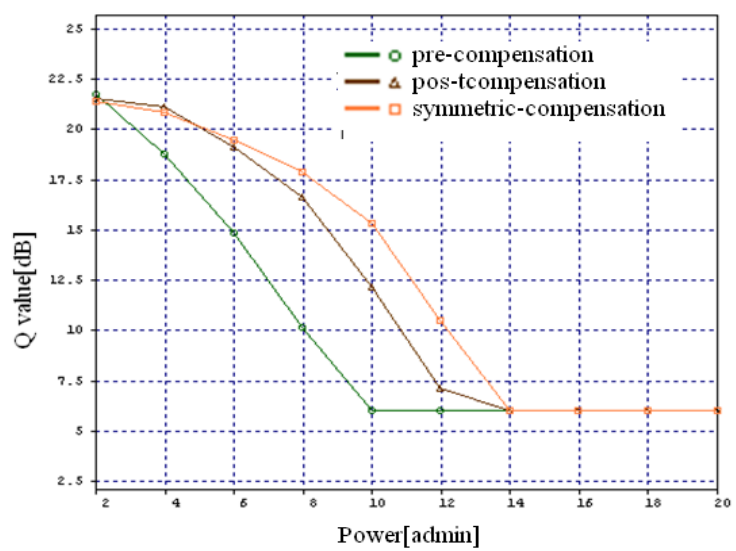

(a)

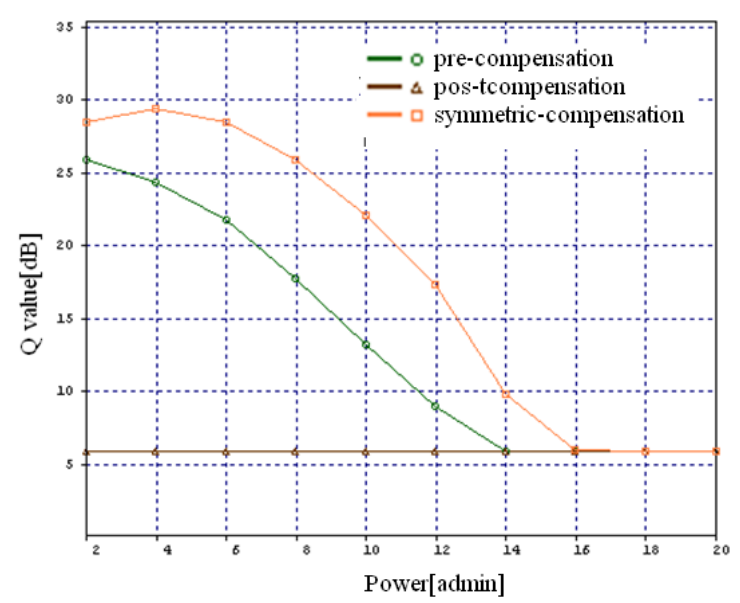

(b)

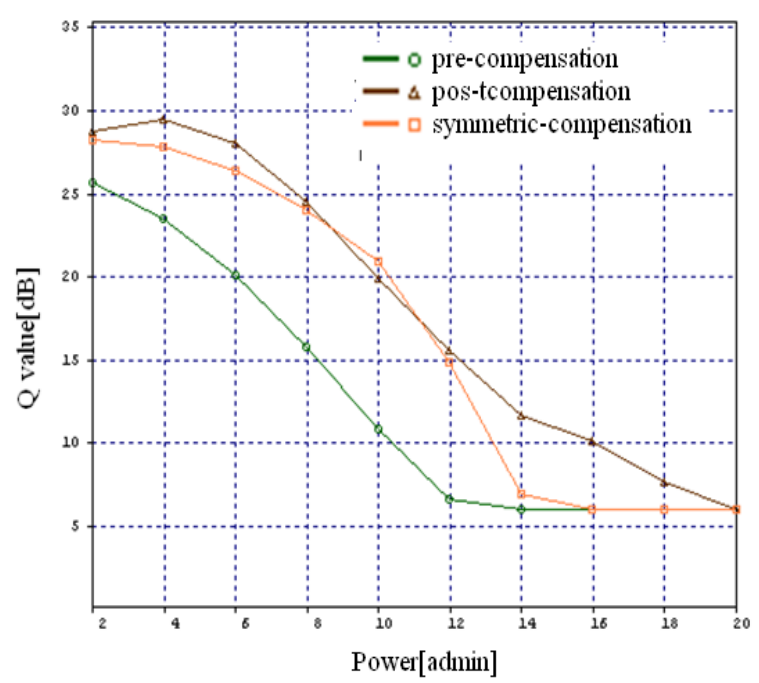

(c)

Fig 2 : Q-parameter Vs power of EDFA with $100 \mathrm{MHz}$ Laser Line width using different DCF compensation Techniques with (a) NRZ Format (b) RZ Format and (c) RZ-Super Gaussian Format at 15Gbps 
For 10Gbps data rate symmetric compensation scheme perform better then pre and post compensation for NRZ modulation format, while for RZ and RZ Supergaussian post compensation scheme perform better then any other schemes up to $12 \mathrm{db}$ power level . Above $12 \mathrm{~dB}$, the performance of all compensation scheme goes down. And for $15 \mathrm{Gbps}$ data rate symmetric compensation perform better for both NRZ and RZ while for RZ super gaussian post compensation scheme perform better up to $14 \mathrm{~dB}$ power level of EDFA.

\subsubsection{Evaluation of Required Optical Power}

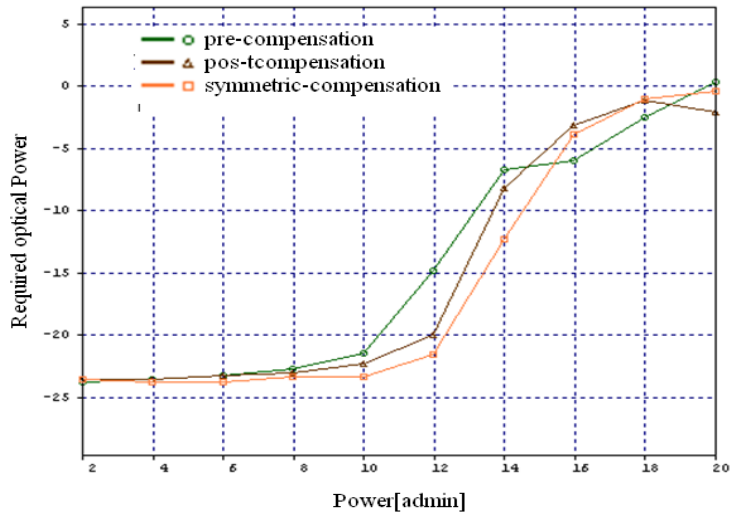

(a)

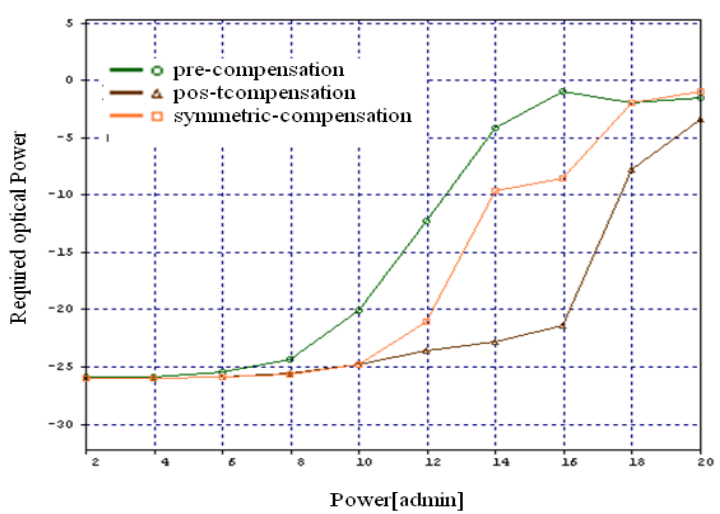

(b)

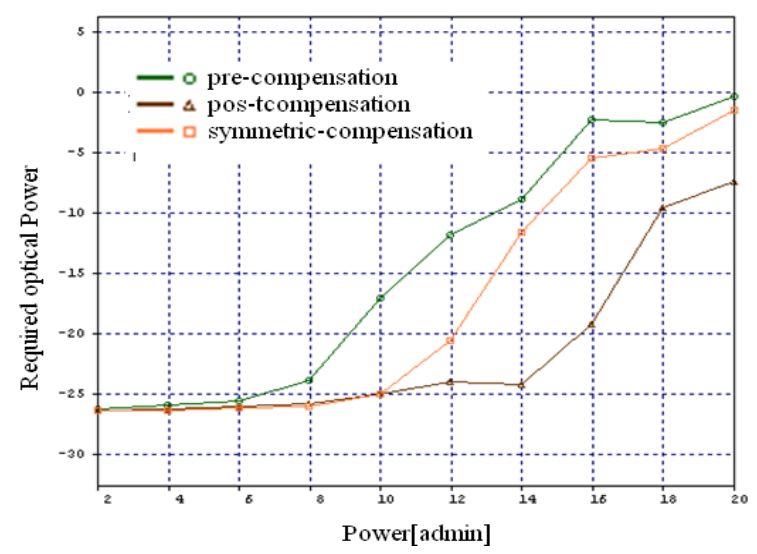

(c)

Fig 3 : Q-parameter Vs power of EDFA with $100 \mathrm{MHz}$ Laser Line width using different DCF compensation Techniques with (a) NRZ Format (b) RZ Format and (c) RZ-Super Gaussian Format at 10Gbps

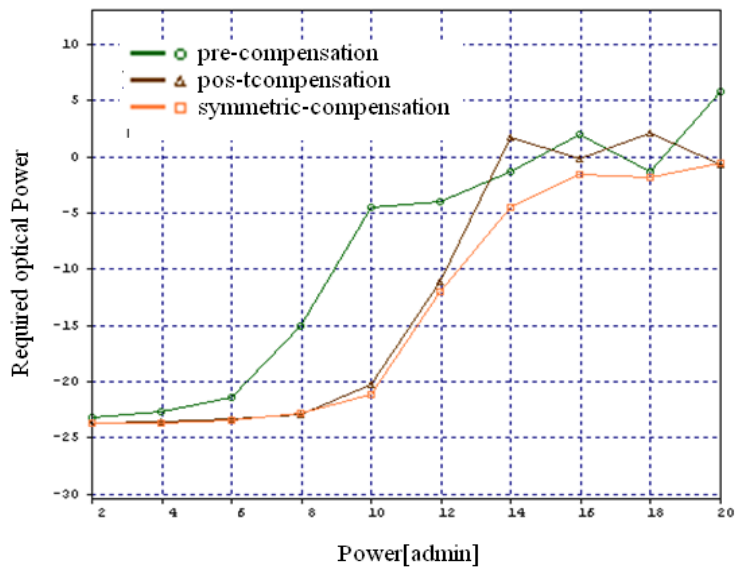

(a)

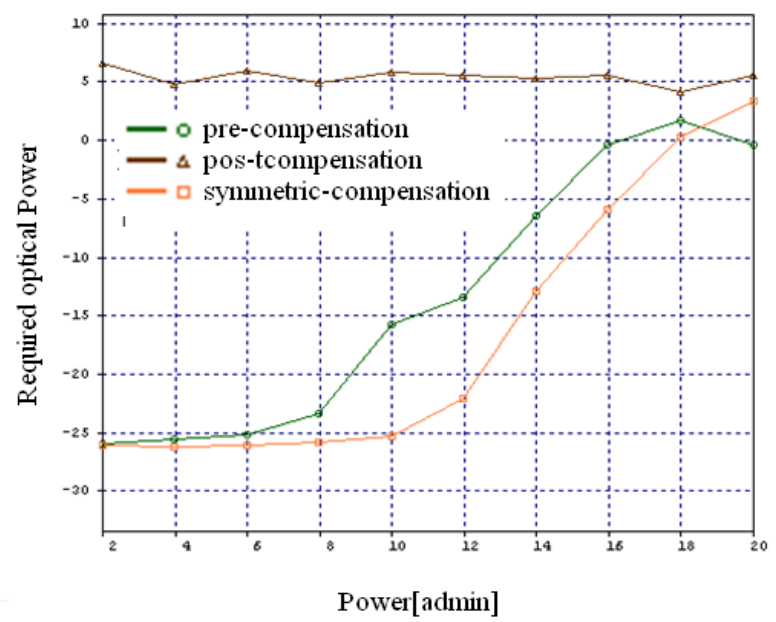

(b)

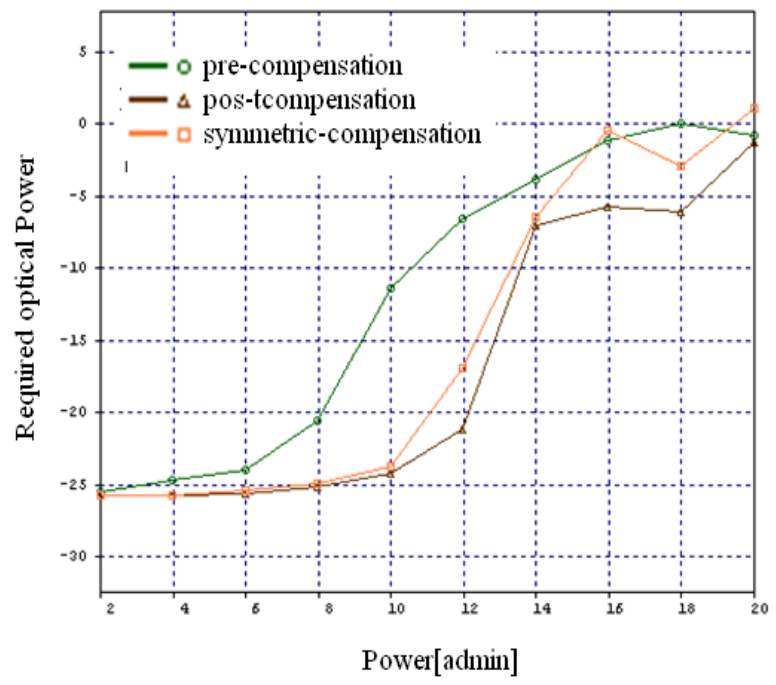

(c)

Fig 4 : Q-parameter Vs power of EDFA with $100 \mathrm{MHz}$ Laser Line width using different DCF compensation Techniques with (a) NRZ Format (b) RZ Format and (c) RZ-Super Gaussian Format at 15Gbps 
From fig we conclude that the graph of optical power is going to increase with the increasing of power of EDFA. We can see that for $10 \mathrm{Gbps}$, for NRZ symmetric compensation scheme perform better up to $12 \mathrm{dBm}$, for $\mathrm{RZ}$ rectangular and RZ super gaussian modulation format postcompensation scheme perform better then any other schemes. While for $15 \mathrm{Gbps}$ symmetric compensation scheme perform better of up to $10 \mathrm{dBm}$ power ,for NRZ and RZ rectangular pulses while for RZ super gaussian pulse post compensation scheme perform better them pre and symmetric compensation scheme up to $10 \mathrm{dBm}$ power of EDFA.

\subsubsection{Evaluation of Bit error rate at optimal} threshold

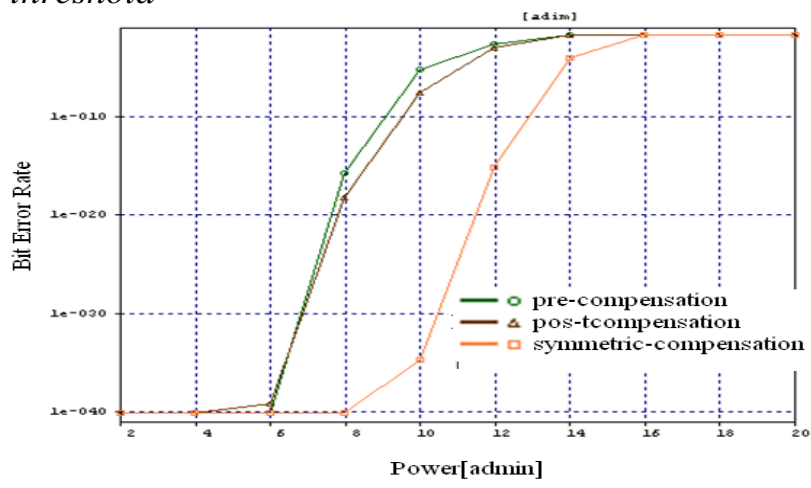

(a)

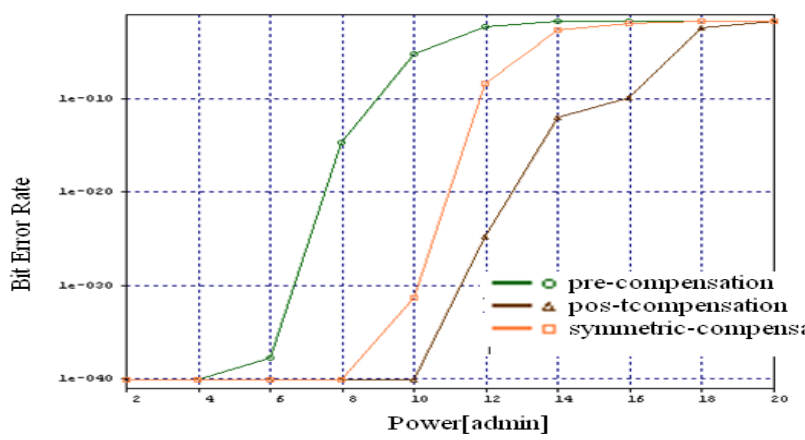

(b)

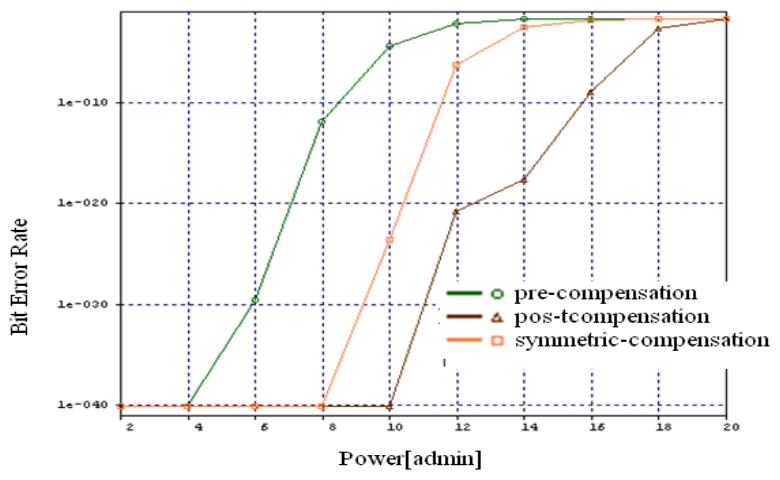

(c)

Fig 5 : Q-parameter Vs power of EDFA with $100 \mathrm{MHz}$ Laser Line width using different DCF compensation Techniques with (a) NRZ Format (b) RZ Format and (c) RZ-Super Gaussian Format at 10Gbps
It is observed that the bit error rate increases with increase in the output power of EDFA. for symmetrical compensation , the Bit error rate is minimum indicating the best performance for NRZ modulation format, for EDFA power up to $8 \mathrm{dBm}$ the Bit error rate is 10-40 but if the power is increase from 10 to 18 the Bit error rate is also increased which is acceptable for high data rate optical transmission. While for RZ and RZ super Gaussian post compensation scheme perform better then pre and symmetric up to $10 \mathrm{dBm}$ power level. In case of $15 \mathrm{Gbps}$ data rate, for NRZ format bit error rate is increasing rapidly from the power of $2 \mathrm{dBm}$ for symmetric compensation, for RZ the bit error rate of post compensation is constant for all power levels of EDFA. And for RZ super Gaussian bit error rate is increase for all compensation schemes when we increase the power from 8 to $12 \mathrm{dBm}$ and above.

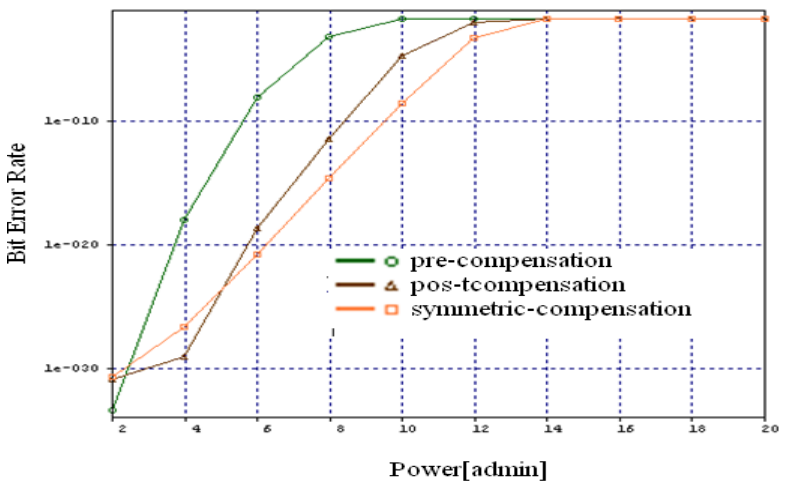

(a)

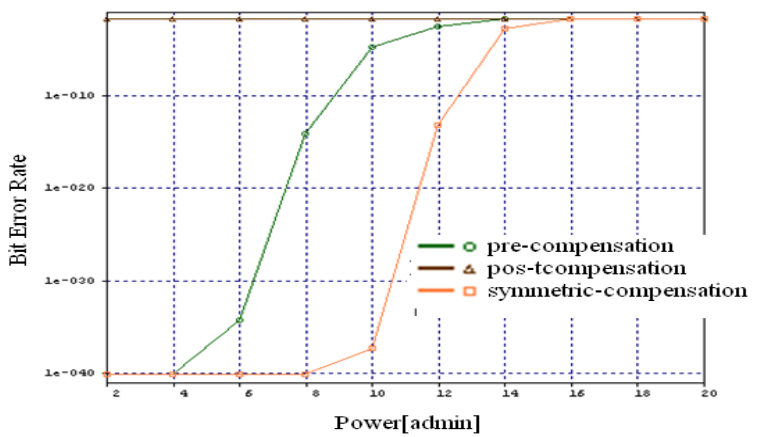

(b)

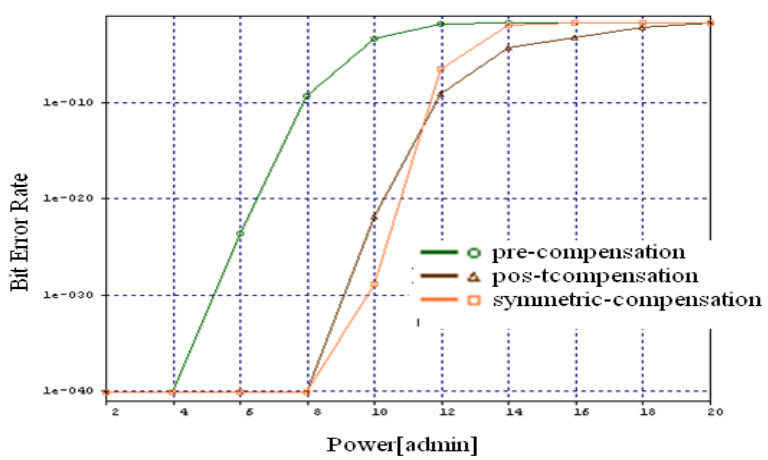

(c)

Fig 6 : Q-parameter Vs power of EDFA with $100 \mathrm{MHz}$ Laser Line width using different DCF compensation Techniques with (a) NRZ Format (b) RZ Format and (c) RZ-Super Gaussian Format at 15Gbps 


\subsection{When Length of Fiber of Each Span and Power of EDFA Both Varied}

\subsection{1 $Q$ parameter evaluation}

In this case when both the power of EDFA and length of fiber is varied ,The Q-parameter of our simulative dispersion compensated optical communication system techniques at 10and 15-Gbps transmission rate at laser line width of $100 \mathrm{MHz}$ is computed. It is seen that the Q-parameter decreases with respect to optical span and power of EDFA. For 10Gbps data rate, NRZ symmetric compensation perform better up to $12 \mathrm{dBm}$ power of EDFA. While for RZ and RZ super Gaussian post compensation scheme perform better. In case of $15 \mathrm{Gbps}$ data rate symmetric compensation scheme perform better then pre and post compensation schemes for all type of modulation format of up to $12 \mathrm{dBm}$ power of EDFA. If we increase power from $12 \mathrm{dBm}$ the performance of all type of compensation schemes will degrade.

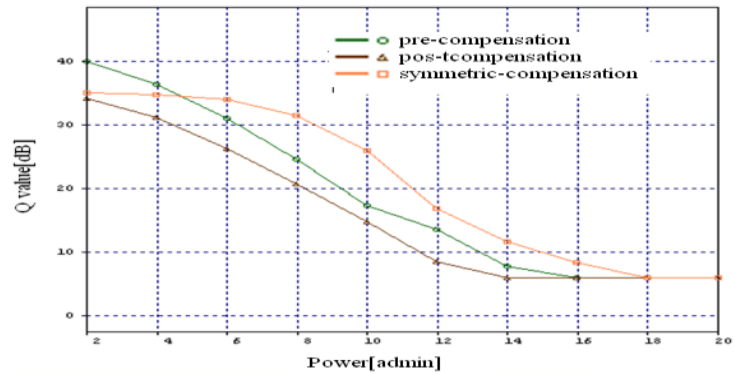

(a)

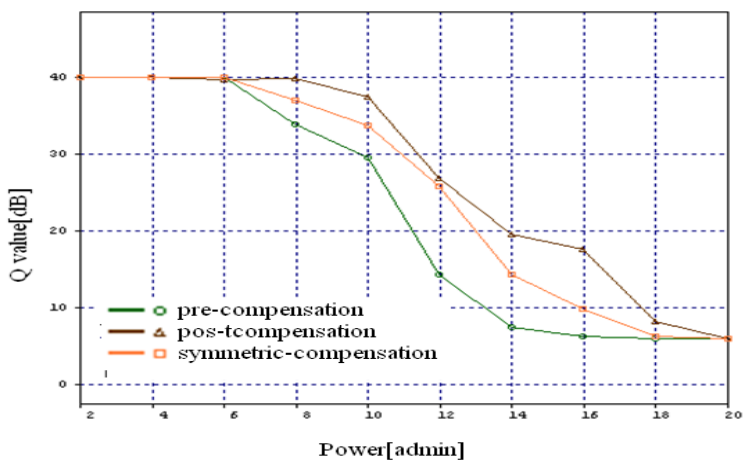

(b)

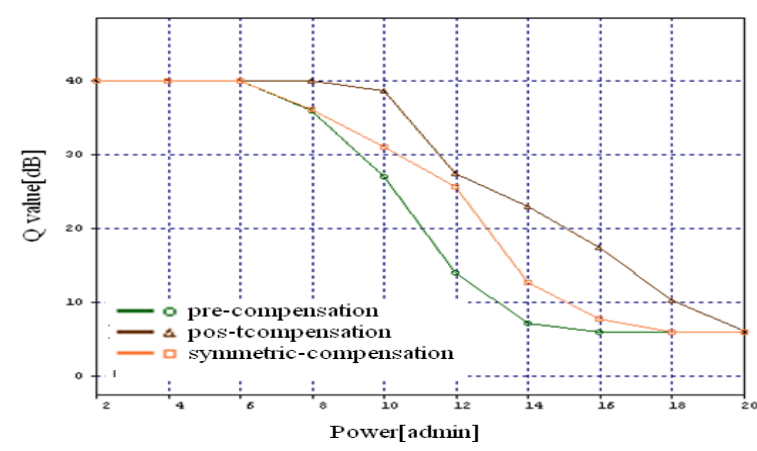

(c)

Fig 7 : Q-parameter Vs power of EDFA with $100 \mathrm{MHz}$ Laser Line width using different DCF compensation Techniques with (a) NRZ Format (b) RZ Format and (c) RZ-Super Gaussian Format at 10Gbps

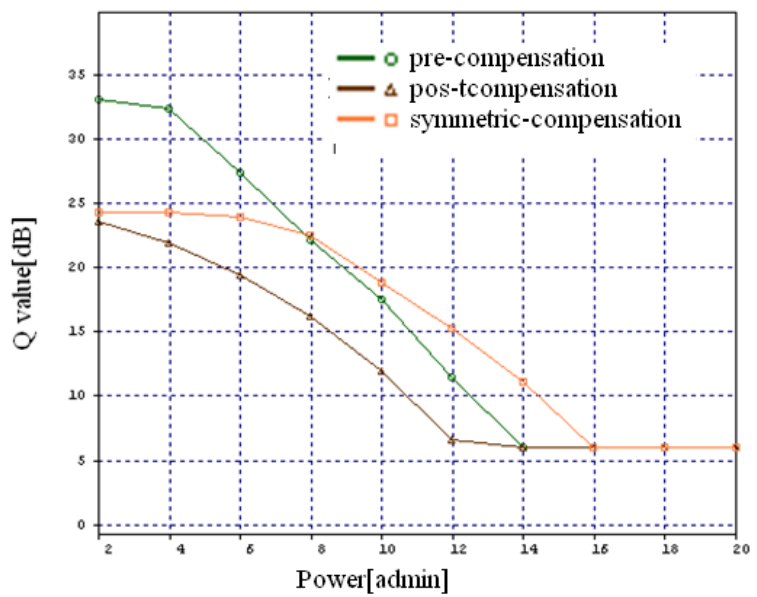

(a)

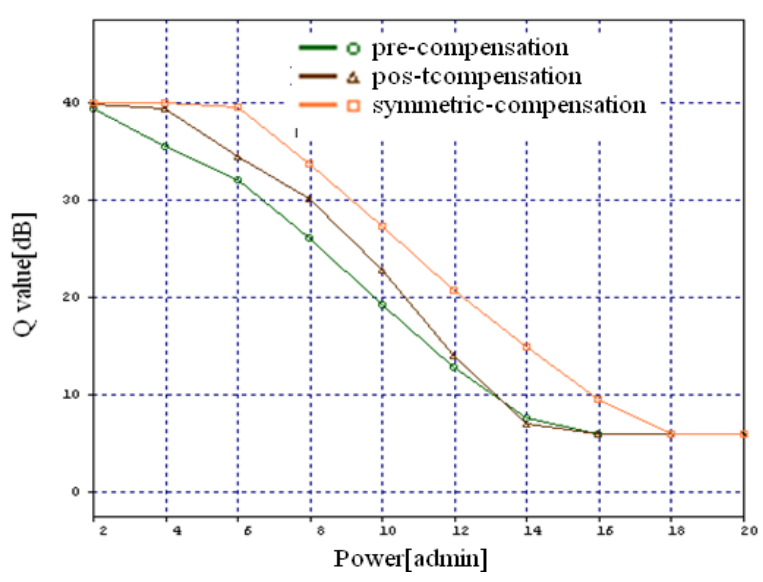

(b)

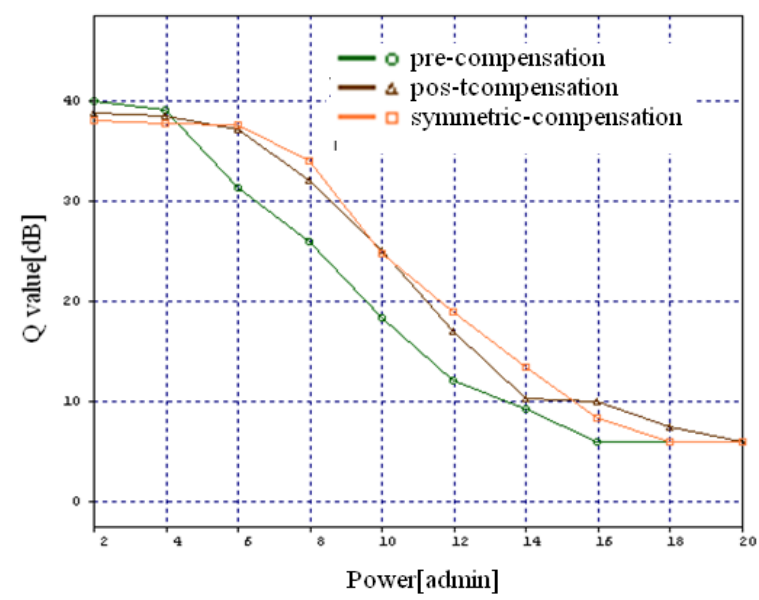

(c)

Fig 8 : Q-parameter Vs power of EDFA with $100 \mathrm{MHz}$ Laser Line width using different DCF compensation Techniques with (a) NRZ Format (b) RZ Format and (c) RZ-Super Gaussian Format at 15Gbps 


\subsubsection{Evaluation of Required Optical Power}

In this case, we investigate the required optical power at sensitivity to compensate the dispersive impact in our simulative dispersion compensated optical communication system after an optical span of $100 \mathrm{Km}$ and with varied power of EDFA, using Pre-, Post- and Symmetric-dispersion compensation techniques at $10-$ and $15-$ Gbps transmission rate at $100 \mathrm{MHz}$ laser line width. It has observed that the required optical power at sensitivity increases with respect to optical span for all the simulated optical pulses and at sharp rate. From fig (a),(b),(c) it is observed that post compensation scheme perform better for RZ and RZ super gaussian optical pulse of up to $14 \mathrm{dBm}$ power level of EDFA, while symmetric and pre compensation perform equally of up to $12 \mathrm{dBm}$ power of EDFA for NRZ optical pulse.

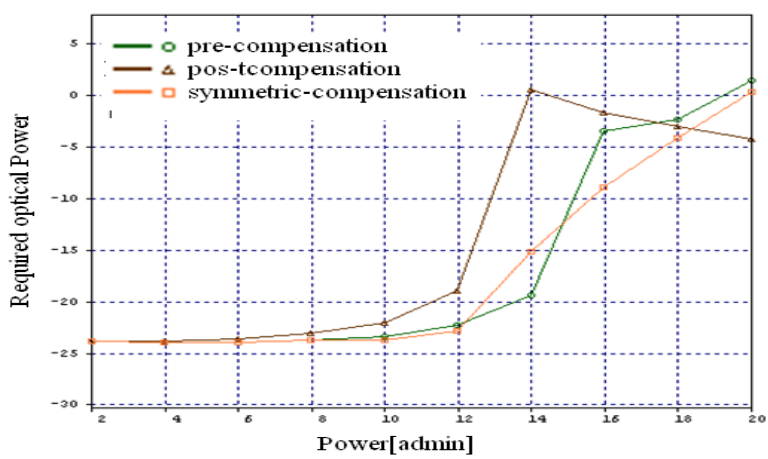

(a)

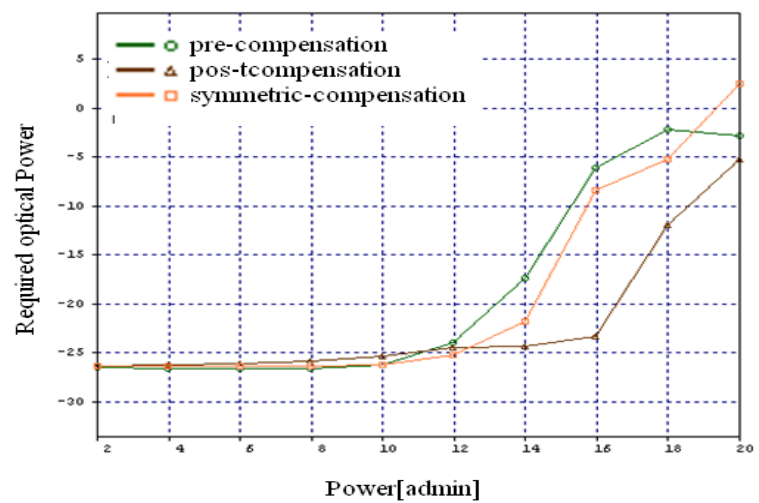

(b)

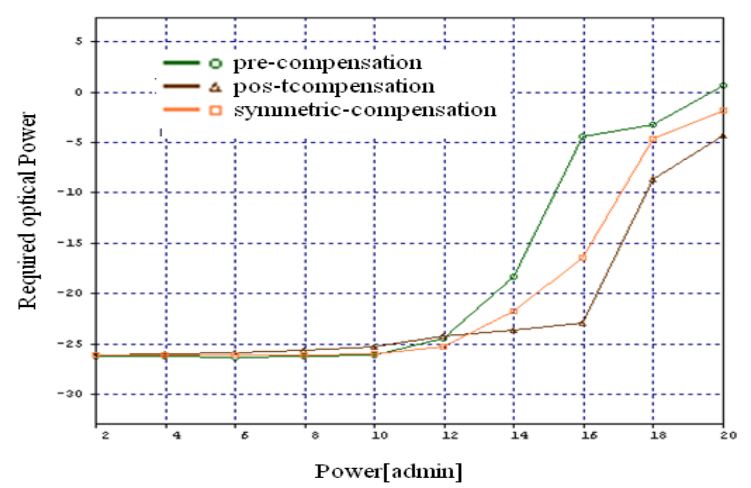

(c)

Fig 9 : Q-parameter Vs power of EDFA with $100 \mathrm{MHz}$ Laser Line width using different DCF compensation Techniques with (a) NRZ Format (b) RZ Format and (c) RZ-Super Gaussian Format at 10Gbps
While in case of $15 \mathrm{Gbps}$ data rate, pre- and post compensation scheme perform equally for NRZ optical pulse and symmetric compensation scheme perform better .of up to $12 \mathrm{dBm}$ power of EDFA. From fig it is conclude that for all type of optical pulse symmetric compensation scheme is better then pre and post compensation scheme of up to $12 \mathrm{dBm}$ power.

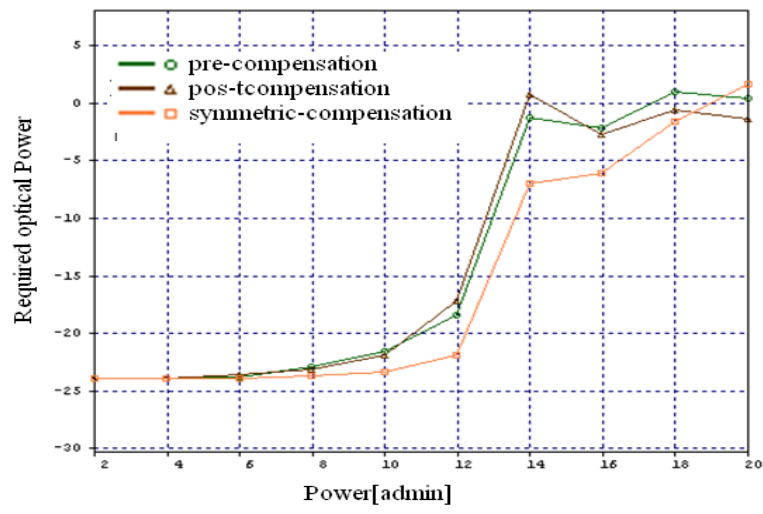

(a)

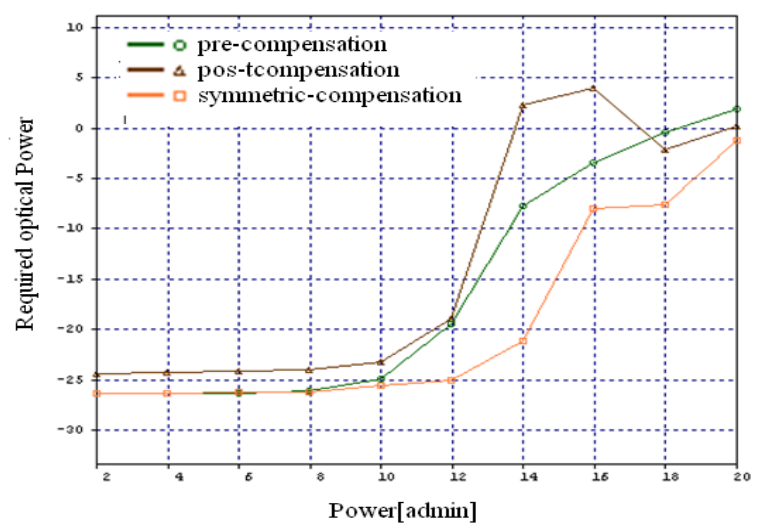

(b)

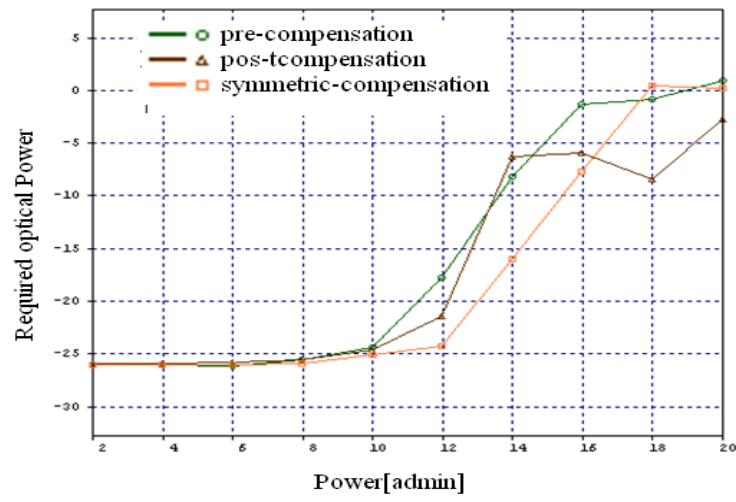

(c)

Fig 10: Q-parameter Vs power of EDFA with $100 \mathrm{MHz}$ Laser Line width using different DCF compensation Techniques with (a) NRZ Format (b) RZ Format and (c) RZ-Super Gaussian Format at 15Gbps 
3.2.3 Evaluation of Bit error rate at optimal threshold
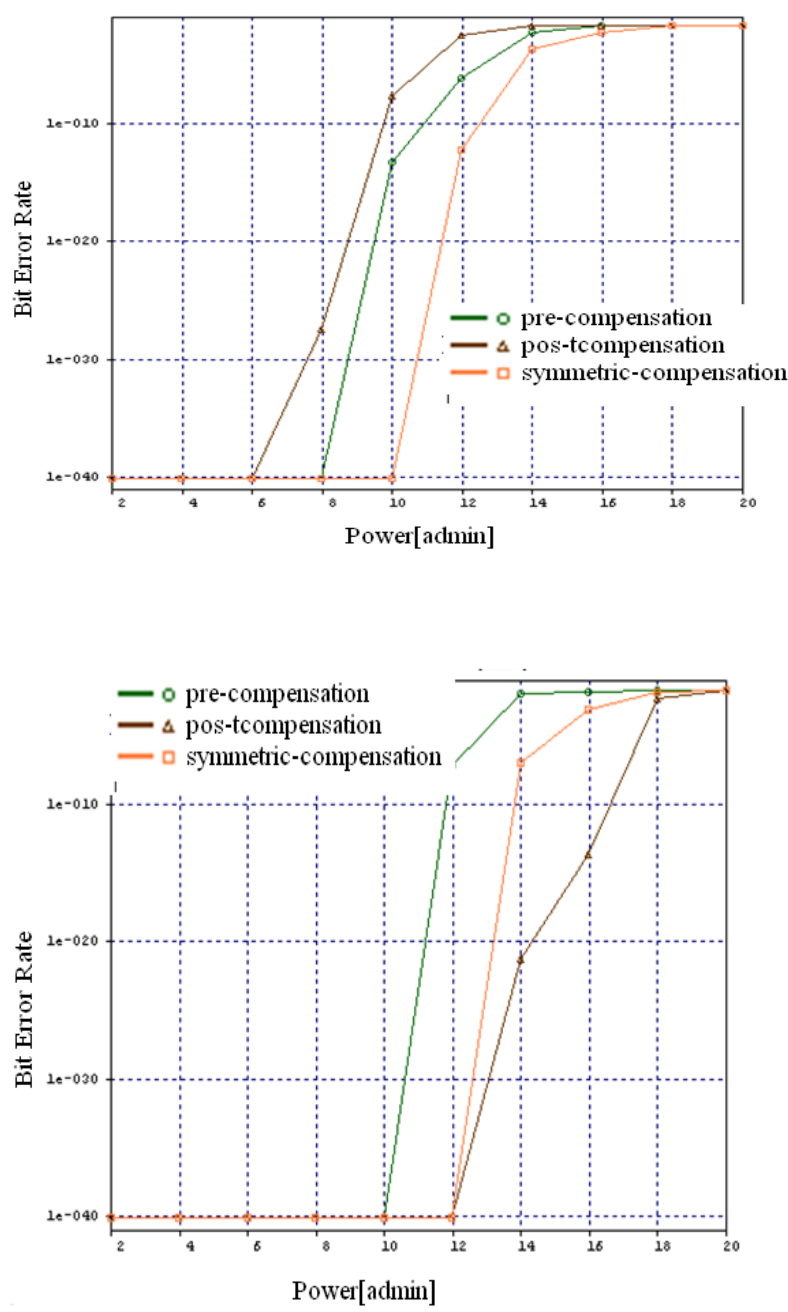

(b)

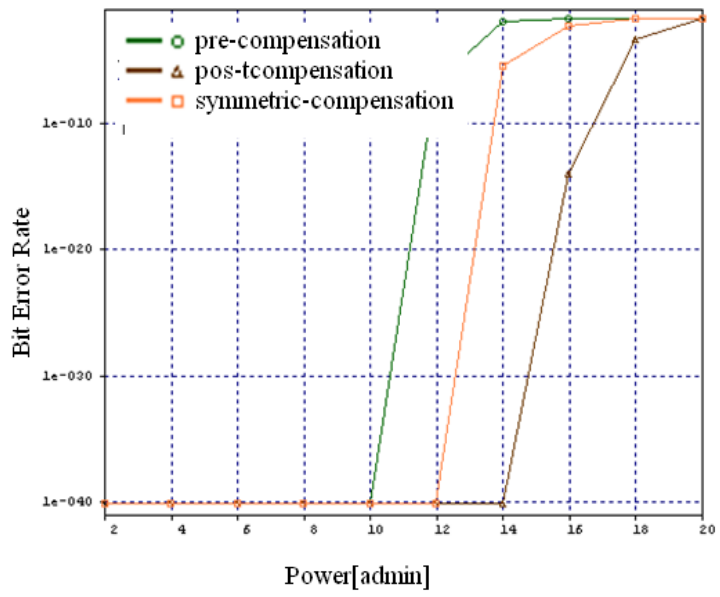

(c)

Fig 11 : Q-parameter Vs power of EDFA with $100 \mathrm{MHz}$ Laser Line width using different DCF compensation Techniques with (a) NRZ Format (b) RZ Format and (c) RZ-Super Gaussian Format at 10Gbps
It is observed that ,for NRZ optical pulse ,the Bit error rate is constant of up to $10 \mathrm{db}$ for all compensation schemes, but when we increase the power of EDFA the Bit error rate is also increasing for symmetric compensation, for RZ modulation format the Bit error rate is constant of up to $12 \mathrm{db}$ power level after this post compensation is better perform ,and RZ super gaussian Bit error is constant of up to $14 \mathrm{db}$ and here also post compensation scheme perform better then pre and symmetric DCF techniques. While in case of $15 \mathrm{Gbps}$ data rate, for all type of modulation formats symmetric compensation scheme perform better then other DCFtechniques ,in case of RZ and RZ super Gaussian bit error rate is constant of up to $10 \mathrm{dBm}$ power of EDFA.

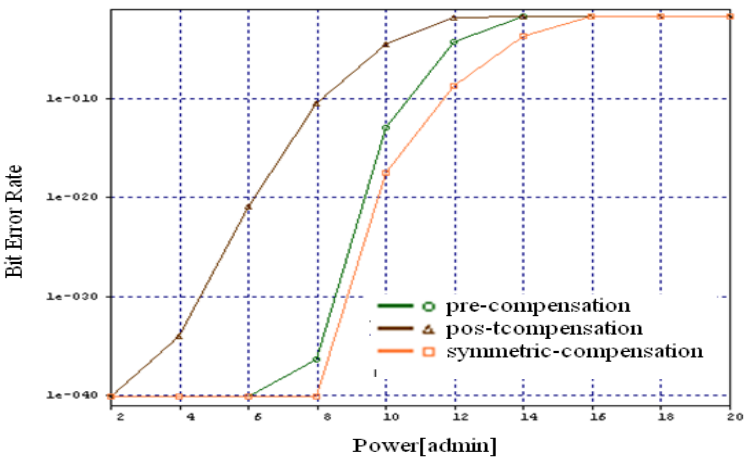

(a)

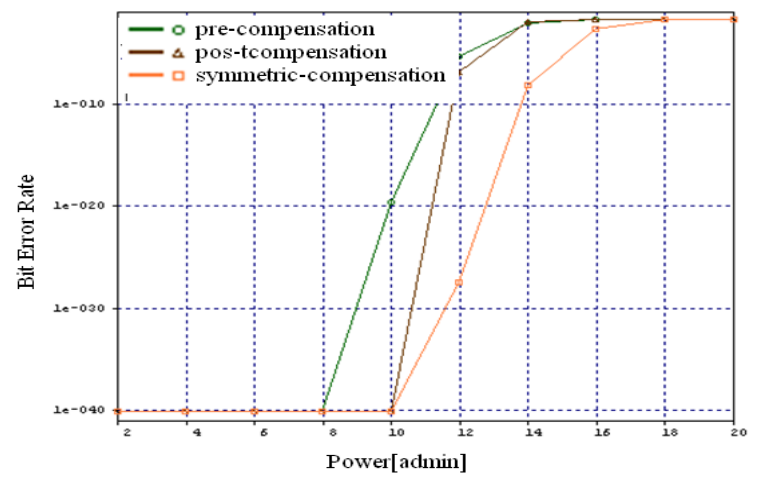

(b)

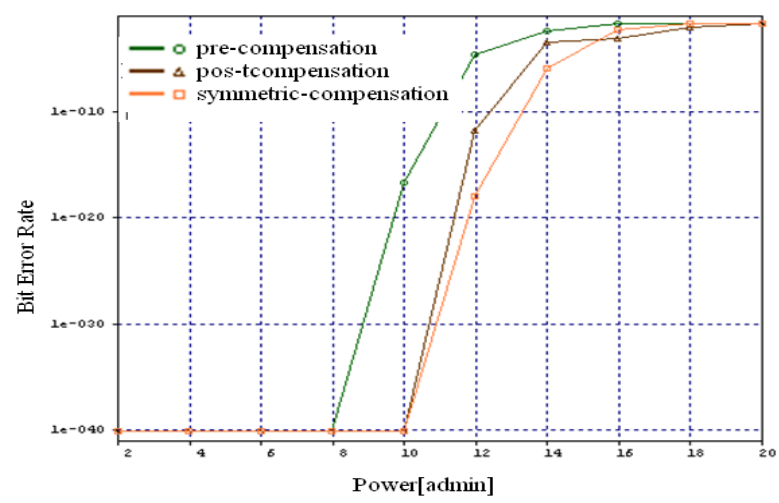

(c)

Fig 12 : Q-parameter Vs power of EDFA with $100 \mathrm{MHz}$ Laser Line width using different DCF compensation Techniques with (a) NRZ Format (b) RZ Format and (c) RZ-Super Gaussian Format at 15Gbps 


\section{CONCLUSION}

In this paper, a detailed investigation of dispersion compensated optical communication system of different optical pulses at 10/15Gbps using pre-, post- and symmetricDCF techniques is reported at Laser line width of $100 \mathrm{MHz}$ for two cases . 3.1 [when only power of EDFA is varied ] and 3.2 [when both power of EDFA and length of optical fiber of each span is varied]. To decrease BER and power penalty required to compensate the dispersion impact in the system, It is recommended to use symmetric- and post-DCF schemes for all the simulated optical pulses rather than using pre-DCF scheme at high transmission rate in dispersion compensated optical communication system in conjunction with laser line width of $100 \mathrm{MHz}$.It conclude that all DCF schemes perform better only of up to $12 \mathrm{dBm}$ power of EDFA. If we increase the power of EDFA from $12-18 \mathrm{dBm}$ only Bit error rate for all type of modulation formats is increases, for $10 / 15 \mathrm{Gbps}$ data rate.

\section{REFERENCES}

[1] K.Tajima, L.M.Washio, Opt.Lett.10 (9) (1985) 460.

[2] T.L. Koch, R.C. Alferness, J. Lightwave Technol. LT3(1985) 800

.[3] R.S. Kaler, T.S. Kamal, A.K. Sharma, S.K. Arya, R.A. Aggarwala, J.Fiber Integrated Opt.21 (3) (2002) 193.

[4] A.H. Gnauck, S.K. Korotky, J.J. Veselka, J. Nagpal, C.T. Kemmerer, W.J. Minford, D.T. Moser, IEEE, Photon. Technol.Lett.3 (10) (1991).

[5] J.J. O'Reilly, M.S. Chauldry, IEEE, Colloquim Microwave Optoelectron.139 (1990) 13/1.

[6] R.M. Jopson, A.H. Gnaules, R.M. Derosier, in: Proc. OFC'93, paper pd3, 1993

[7] A.Yariv, D.Feke, D.M.Pepper, Opt.Lett.4 (1979) 52.

[8] S.Watanable, T.Naito, T. Chikma, IEEE, Photon. Technol.Lett.5 (1) (1993) 92

[9] C.D. Poole, J.M. Wieserfed, D.J. DiGiovanni, A.M.Vengsarkar, J.Lightwave Technol. 12 (10) (1994) 1746.

[10] R.-D. Li, P. Kumar, W.L. Kath, J. Lightwave Technol. 12 (3) (1994) 541

[11] J.C. Cartledge, H. Debregeans, C. Rolland, IEEE, Photon. Technol.7 (2) (1995) 224.

[12] N.Henmi, T. Saito, T.Ishida, J.Lightwave Technol. 12 (10) (1994) 1706.

[13] A.J. Antos, D.K. Smith, J. Lightwave Technol. 12 (10) (1994) 1739.

[14] A.Djupsjobak a, O.Sahlen, IEEE, J. Lightwave Technol. 12 (10) (1994) 1849.

[15] A.K. Sharma, R.K. Sinha, R.A. Agarwala, J. Fiber Integrated Opt.16 (4) (1997).

[16] A.K. Sharma, R.K. Sinha, R.A. Agarwala, J. Opt. Fiber Technol.4 (1998) 135
[17] A.K. Sharma, R.K. Sinha, R.A. Agarwala, Int. J. Light Electron Opt., Optik 111 (7) (2000) 310.

[18] G.P. Aggrawal, Fiber Optic Communication Systems, wiley, New York, 1997.

[19] C.Peuchere t, N.Hanik, R.Freund, L.Molle, P.Jeppesen IEEE Photon.Technol.Lett.12 (8) (2000) 992

[20] R.J. Nuyts, Y.K. Park, P. Gallison, Photon. Technol. Lett. 8 (1996) 1406

[21] D.M. Rothnie, J.E. Midwinter, Electron. Lett. 32 (20)(1996) 1907

[22] G.P. Aggrawal, Nonlinear Fiber Optics, Academic Press,San Deigo, CA, 1995.

[23] A.H. Gnauck, S.K. Korotky, J.J. Veselka, J. Nagpal, C.T. Kemmerer, W.J. Minford, D.T. Moser, IEEE Photonics Technology Letters, Volume 3, Issue 10, 1991

[24] C.D. Poole, J.M. Wieserfed, D.J. DiGiovanni, A.M. Vengsarkar, Journal of Light wave Technology, Volume 12, Issue 10, 1994, 1746.

[25] A.J. Antos, D.K. Smith, Journal of Light wave Technology, Volume 12, Issue 10, 1994, 1739.

[26] A.K. Sharma, R.K. Sinha, R.A. Agarwala, Optik, Volume 111, Issue 7, 2000, 310

[27] C.Peuchere t, N.Hanik, R.Freund, L.Molle, P.Jeppesen , IEEE Photonics Technology Letters, Volume 12, Issue 8 , 2000, 992.

[28] Manjit singh , Ajay K. Sharma, R.S.Kaler , “ Investigations on order and width of RZ super Gaussian pulse in pre-, post-,and symmetrical dispersion compensated $10 \mathrm{~Gb} / \mathrm{s}$ optical communication system using standard and dispersion compensated fiber", volume 121, Issue 7 , 2010, pg-609-616

[29] Monaj kumar, Ajay K. Sharma, T.S.Kamal , etc, “ Comparative investigation and suitability of various data formats for $10 \mathrm{~Gb} / \mathrm{s}$ optical Soliton transmission links at different chirps", volume120, Issue7, March 2009, pg330-336

[30] R.S.Kaler, Ajay K. Sharma, T.S.Kamal, "compression of pre-, post- and symmetrical dispersion compensation schemes for $10 \mathrm{~Gb} / \mathrm{s}$ NRZ links using standard and dispersion compensated fibers", Optics Communication 209,2002,pg-107-123

[31] Juanjuan Yan, Minghua Chen , Shizhong Xie,Bingkun Zhou, "Performance comparison of standard FEC in 40 $\mathrm{Gb} / \mathrm{s}$ optical transmission system with NRZ, RZ and CSRZ modulation formats" , Optics Communication , volume 231, feb.2004.pg- 175-185 